ISSN 2447-9071

doi $10.36414 /$ rbmc.v5i12.11
Contato para correspondência: Karlla Greick Batista Dias Penna

E-mail:

karllagreick@gmail.com

Conflito de interesse: Não

Financiamento: Recursos próprios

Recebido: 28/06/2019

Aprovado: 13/08/2019

\section{As diretrizes curriculares nacionais dos cursos de graduação da área da saúde abordam as políticas públicas e o sistema único de saúde?}

\section{Do national curriculum guidelines for health graduation courses address public policies and the single health system?}

Frank Sousa Castro', Alesssandra Marques Cardoso², Karlla Greick Batista Dias Penna ${ }^{3}$

'Biomédico, Mestre em Ciências Ambientais e Saúde pela PUC Goiás, Professor da Faculdade da Polícia Militar, ${ }^{2}$ Biomédica, Doutora em Medicina Tropical e Saúde Pública pela UFG, Professora da Faculdade da Polícia Militar, ${ }^{3}$ Biomédica, Doutora em Biologia Celular e Molecular pela UFG, Coordenadora do curso de Biomedicina da PUC Goiás.

\section{Resumo}

A Política de Educação em Saúde objetiva a luta coletiva para que a formação dos trabalhadores da saúde se torne uma prática para o Sistema Único de Saúde um compromisso do Estado Brasileiro, no sentido de contribuir para transformar o modelo de atenção, consolidando as políticas públicas. As profissões em saúde merecematenção não sópela importância em ampliaro conhecimento científico como também em análises do modo de pensar e agir dos sujeitos definidos neste contexto. Essa preocupação torna-se evidente com o Parecer Técnico n 300/2017, desenvolvido a partir da 286a Reunião Ordinária, sendo que a Conselho Nacional Saúde aprovou a criação de um Grupo de Trabalho para discutir as Diretrizes Curriculares Nacionais (DCN) dos cursos de graduação da saúde, na perspectiva de que possam expressar os princípios e as necessidades do SUS. Assim esta pesquisa foi realizada a partir do levantamento das DCN das 14 profissões em saúde, buscando de forma ativa, usando como fonte de dados para identificação os descritores Políticas Públicas e SUS. Das 14 profissões, 10 não mencionam o termo SUS, enquanto que o termo Políticas Públicas também não é mencionado em outras profissões. A partir da observação das DCN dos cursos Farmácia (2017) e Medicina (2014), recentemente estruturadas, fica claro que estes cursos articulam bem em suas competências e habilidades o futuro profissional em saúde a dinâmica das polícias públicas do SUS. Assim vislumbrar essa aplicação nas DCN de todos os cursos da saúde a partir da inserção nos currículos e programasé possível a construção de estruturas curriculares que compõe os PPC destas profissões.

Palavras-Chave: Educação em Saúde, Políticas Públicas de Saúde, Sistema Único de Saúde, Diretrizes Curriculares Nacionais.

\begin{abstract}
The Health Education Policy starts to have the objective of collective struggle in the health area so that the formation of workers becomes a practice for the SUS is a commitment of the Brazilian State, in the sense of contributing to transform the attention model, consolidating the Public policy. Health professions deserve a lot of attention, not only because of the importance they acquire in broadening scientific knowledge but also in analyzes of the way of thinking and acting of the subjects defined therein. This concern becomes evident with Technical Opinion no. 300/2017, which was developed from the 286th Ordinary Meeting, and the CNS approved the creation of a Working Group to discuss the National Curricular Guidelines (CND) of undergraduate health courses, in the perspective that can express the principles and needs of SUS. In this way the research was done from the survey in the DCN of the 14 professions in health, searching actively, using as initial source of data for identification the words Public Policies andSUS. Of the 14 professions, 10 professions donotmention the wordSUS, while
\end{abstract}


Public Policies are also not mentioned in 10 professions. Looking at the recently created Pharmacy (2017) and Medicine (2014) DCNs, it is clear that these two courses articulate well in their skills and abilities the future professional in health the dynamics of the Public Police of the SUS. Thus, to see this application in the DCN of all health courses comes to change curricula and programs, making possible the construction of curricular structures that make up the PPC of these courses.

Keywords: DCN, Public Policy, SUS, CNS, CNE, Education.

\section{Introdução}

"A saúde é direito de todos e dever do Estado, garantido mediante políticas sociais e econômicas que visem à redução do risco de doença e de outros agravos e ao acesso universal e igualitário às ações e serviços para sua promoção, proteção e recuperação"'.

Baseado no art. 196 da Constituição Federal de 1988, definir saúde na origem etimológica da palavra como atributo principal dos inteiros, íntegros, e no grego salus provém do termo holos, no sentido de totalidade, raiz dos termos holismo, holístico. Ou seja, este termo refere-se ao todo. Indaga-se o que passa ser o todo para um país que apresenta dimensões continentais. Todos os brasileiros têm acesso à saúde, na mesma ótica todos os profissionais envolvidos nesta política podem promover a saúde da população².

A Organização Mundial de Saúde (OMS), no ano de 1946 definiu saúde como "um estado de completo bem estar físico, mental e social, e não consiste apenas na ausência de doença ou de enfermidade"

Uma vez definido o termo saúde, se faz necessário compreender a sua evolução histórica, religiosa e principalmente a sua aplicação de forma humanizada para toda a população, através das políticas públicas de saúde.

Lucchese, em 2002, tentou definir Políticas Públicas, "como conjunto de disposições, medidas e procedimentos que traduzem as diretrizes ou deveres das políticas do Estado e regulam as atividades governamentais relacionadas às tarefas de interesse público". As políticas públicas de saúde fazem parte das ações do Estado orientado para a melhoria das condições de saúde da população organizando as funções públicas governamentais para a promoção, proteção e recuperação da saúde dos indivíduos e da coletividade 3 .

Com a promulgação da constituição federal de 1988, aparentemente ocorreu a garantia da efetivação das políticas públicas de saúde como um direito universal e igual para todos, além de promover uma descentralização da gestão entre seus entes federados ${ }^{4}$. Só que essa conquista não partiu unicamente da classe política, mas sim dos movimentos sociais e populares que defenderam o direito à saúde como um direito vinculado à cidadania, que propunham um sistema de saúde de caráter público, sob a responsabilidade do Estado.
Nota-se que as bases do atual Sistema Único de Saúde (SUS)

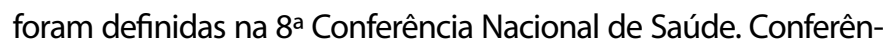
cia que foi realizada em março de 1986 e produziu um relatório que subsidiou decisivamente a Constituição Federal de 1988 nos assuntos de Saúde, "ensejando mudanças baseadas no direito universal à saúde, acesso igualitário, descentralização acelerada e ampla participação da sociedade ${ }^{\prime \prime 5}$.Sendo necessário incluir as leis que regulamentam as políticas públicas de saúde no Brasil, como a Lei Orgânica da Saúde (Lei 8080/90), a lei complementar da Saúde (Lei 8142/90), a Lei Orgânica da Seguridade Social (Lei 8212/91) e a Lei Orgânica da Assistência Social (Lei 8742/93). Ainda referindo à saúde deve se citar a Emenda Constitucional n 29 de 13 de setembro de 2000, que passa a "definir a participação das esferas federal, estadual e municipal de governo no financiamento das ações e serviços públicos de saúde" ${ }^{\prime \prime}$.

OSUS éo sistema hegemônico do país, sendo responsável pela atenção à saúde da maioria dos cidadãos brasileiros. Formado por um conjunto de serviços ambulatoriais especializados; serviços de apoio ao diagnóstico e a terapêutica; serviço pré-hospitalar de urgência e emergência, com destaque para o Serviço Móvel de Urgência (SAMU); além de uma rede hospitalar de média e de alta complexidade. Compondo um conjunto que reúne ações de promoção, proteção e recuperação da saúde, bem como, das ações prevenção de doenças e agravos ${ }^{6}$.

Além de ofertar serviços de infraestrutura à população brasileira, seguindo constituição de 1988, o SUS nos termos do art. 200, inciso III, obrigação de, "ordenar a formação de recursos humanos na área de saúde", que está também respaldada no art. $6^{\circ}$ da Lei $n^{\circ} 8.080$, de 19 de setembro de 1990. Percebe se que a formação de profissionais de saúde para o SUS ainda está muito distante do desejado.

O perfil dos profissionais de saúde demonstra ainda seguindo as Diretrizes Curriculares Nacionais (DCN) de forma insuficiente copetentes, para as mudanças das práticas nas atividades profissionais. Sendo que as demandas nos serviços de saúde exigem uma crescente necessidade de educação permanente para os profissionais, com o objetivo de implantação e fortalecimento da atenção à saúde no SUS. 
A Política de Educação em Saúde passa a ter um objetivo de luta coletiva na área da saúde para que a formação dos trabalhadores se torne uma prática para a política pública e um compromisso do Estado brasileiro, no sentido de contribuir para transformar o modelo de atenção, consolidando o SUS.

As profissões da saúde merecem muita atenção, não só pela importância que adquirem de ampliar o conhecimento científico como também em análises do modo de pensar e agir dos sujeitos que aí são definidos no contexto do SUS. Formar profissionais com visão futura para o SUS é um desafio, com a finalidade de melhorar a vida das pessoas, ampliando as alternativas apropriadas às mudanças da realidade e colaborando com o desenvolvimento dos trabalhadores da saúde.

Em 2017, o Plenário do Conselho Nacional de Saúde (CNS), em sua Trecentésima Reunião Ordinária, realizada nos dias 7 e 8 de dezembro de 2017, e no uso de suas competências regimentais e atribuições conferidas pela Lei n 8.080 , de 19 de setembro de 1990; pela Lei $n^{\circ} 8.142$, de 28 de dezembro de 1990; pela Lei Complementar no 141, de 13 de janeiro de 2012; pelo Decreto no 5.839, de 11 de julho de 2006; cumprindo as disposições da Constituição da República Federativa do Brasil de 1988, da legislação brasileira correlata: Resolve aprovar, Resolução n 569 de 8 de dezembro de 2017 (Quadro 1):

Quadro 1. Incisos aprovados na Resolução № 569, de 08 de dezembro de 2017.

\footnotetext{
I- Defesa da vida e defesa do SUS como preceitos orientadores do perfil dos egressos da área da saúde;

II - Atendimento às necessidades sociais em saúde;

III - Integração ensino-serviço-gestão-comunidade;

IV - Integralidade e Redes de Atenção à Saúde (RAS);

V - Trabalho interprofissional;

$\mathrm{VI}$ - Projetos Pedagógicos de Cursos (PPC) e componentes curriculares coerentes com as necessidades sociais em saúde;

VII - Utilização de metodologias de ensino que promovam a aprendizagem colaborativa e significativa;

VIII-Valorização da docência na graduação, do profissional da rede de serviços e do protagonismo estudantil;

IX - Educação e comunicação em saúde;

X - Avaliação com caráter processual e formativo;

XI - Pesquisas e tecnologias diversificadas em saúde;

XII - Formação presencial e carga horária mínima para cursos de graduação da área da saúde;
}

Fonte: http://conselho.saude.gov.br/resolucoes/2017/Reso569.pdf. Acesso em 10 de abril de 2018.(8)
Art. $1^{\circ}$ Reafirmar a prerrogativa constitucional do SUS em ordenar a formação dos (as) trabalhadores (as) da área da saúde.

Art. $2^{\circ}$ Aprovar o Parecer Técnico n 300/2017, que apresenta princípios gerais a serem incorporados nas $D C N$ de todos os cursos de graduação da área da saúde, como elementos norteadores para o desenvolvimento dos currículos e das atividades didático-pedagógicas, e que deverão compor o perfil dos egressos desses cursos.

Art. $3^{\circ}$ Aprovar os pressupostos, princípios e diretrizes comuns para a graduação na área da saúde, construídos na perspectiva do controle/participação social em saúde, e apresentados, sinteticamente, nos incisos conforme Quadro 1.

Verifica-se uma preocupação crescente pelos conselhos profissionais de classe com as mudanças na formação profissional em saúde, na qual cada vez mais se configura a necessidade de profissionais aptos a contribuir com a sociedade no contexto do SUS e não somente em seu próprio campo profissional, mas também nos campos político, cultural e social 7 .

Essa preocupação torna evidente com o Parecer Técnico n 300/2017, que foi desenvolvido a partir da 286a Reunião Ordinária (RO) CNS, ocorrida em 6 e 7 de outubro de 2016, o CNS que aprovou a criação de um Grupo de Trabalho (GT/DCN) para discutir as Diretrizes Curriculares Nacionais (DCN) dos cursos de graduação da área da saúde, na perspectiva de que possam expressar os princípios e as necessidades do SUS, com base na já citada Resolução CNS n 350/2005, para assegurar a integralidade da atenção, a qualidade e a humanização do atendimento prestado aos indivíduos, famílias e comunidades. Este (GT/DCN) foi formado por cinco Conselheiros Nacionais de Saúde, representantes dos segmentos de usuários, trabalhadores e gestores do SUS.

O GT/DCN reuniu-se para tratar das profissões em saúde, pautado no processo de construção coletiva com a perspectiva da articulação entre gestão, atenção, educação e trabalho em saúde, uma vez que os serviços públicos integrantes do SUS constituemse como campo de prática para o ensino, a pesquisa e a extensão, mediante normas explicitadas no sistema educacional. Essa formação profissional deve estar intrinsecamente relacionada com a atuação profissional, "entendemos o trabalho no SUS como lócus produtor do conhecimento, um espaço de ensino-aprendizagem".

A formação em serviço proporciona não somente a qualificação dos trabalhadores do SUS, mas o desenvolvimento do próprio sistema de saúde, partindo da reflexão sobre a realidade dos serviçose sobre o que precisa ser transformado, com a finalidade de melhorar a gestão e o cuidado em saúde. Institui-se de forma fundamental que as DCN retratem a produção social da saúde, reafirmem que os trabalhadores da área da saúde estão inseridos nesse processo como agentes transformadores da sociedade, visando garantir saúde para a população através das políticas do SUS9. 
Nosso objetivo nesta pesquisa, visa fomentar aos leitores indagações sobre como esta formação de profissionais de saúde para o SUS relacionado com as competências e habilidades ofertadas nas ultimas DCN.

\section{Métodos}

A pesquisa foi construída a partir do levantamento exclusivo nas DCN das 14 profissões em saúde definidas pela Resolução 287/98 CNS em 08/10/1998. Realizou-se a busca ativa nas DCN pela menção de políticas de saúde do SUS, usando como fonte inicial de dados para identificação os descritores políticas públicas e SUS.

Os procedimentos de busca utilizados ocorreram em dois sentidos complementares. Primeiramente, foi feita a busca nas 14 DCN dos cursos da saúde dentro dos incisos, artigos e parágrafos o descritor SUS, ordenamento e contagem dos mesmos, o que permitiu estabelecer alguns dados. Em um segundo momento, realizou-se a busca ativa por políticas públicas, inseridas nas DCN.

\section{Resultados e Discussão}

Resultados semelhantes foram observados em todos os 14 cursos, tanto para busca de políticas públicas quanto para o SUS. O Quadro 2 sumariza os achados.

Quadro 2. Resultados obtidos a partir da análise das DCN quanto ao SUS e às políticas públicas.

\begin{tabular}{|c|c|c|c|}
\hline $\begin{array}{l}\text { Cursos de Graduação } \\
\text { da Área da Saúde }\end{array}$ & DCN & SUS & $\begin{array}{l}\text { Políticas } \\
\text { Públicas }\end{array}$ \\
\hline Biomedicina & $\begin{array}{l}\text { RESOLUÇÃO CNE/ } \\
\text { CES 2, DE } 18 \text { DE FE- } \\
\text { VEREIRO DE } 2003\end{array}$ & Não menciona & Não menciona \\
\hline Ciências Biológicas & $\begin{array}{c}\text { RESOLUÇÃO CNE/CES } \\
\text { 7, DE } 11 \text { DE MARÇO } \\
\text { DE 2002.(*) }\end{array}$ & Não Menciona & Não Menciona \\
\hline Educação Física & $\begin{array}{l}\text { RESOLUÇÃO CNE/ } \\
\text { CES N 7, DE } 31 \text { DE } \\
\text { MARÇO DE } 2004\end{array}$ & Não Menciona & $\begin{array}{l}\text { Menciona, } \\
\text { Art. } 6^{\circ}: \S 1^{\circ}\end{array}$ \\
\hline Enfermagem & $\begin{array}{l}\text { RESOLUÇÃO CNE/ } \\
\text { CES N³, DE } 7 \text { DE NO- } \\
\text { VEMBRO DE } 2001\end{array}$ & $\begin{array}{l}\text { Menciona, } \\
\text { Art. 5०: XXXIII }\end{array}$ & Não Menciona \\
\hline Farmácia & $\begin{array}{l}\text { RESOLUÇÃO CNE/ } \\
\text { CES N 6, DE } 19 \text { DE } \\
\text { OUTUBRO DE } 2017\end{array}$ & $\begin{array}{c}\text { Menciona, } \\
\text { Art. } 4^{\circ}: \bigvee \\
\text { Art. } 7^{\circ}: \text { III - } \S 4^{\circ} \\
\text { Art. } 12 \S 2^{\circ}\end{array}$ & $\begin{array}{c}\text { Menciona Art. } \\
\text { 4: } \mathrm{VI} \\
\text { Art. } 5^{\circ}: \S 2^{\circ} \\
\text { XVII - } \S 6^{\circ} \mathrm{A}\end{array}$ \\
\hline Fisioterapia & $\begin{array}{l}\text { RESOLUÇÃO CNE/CES } \\
\text { 4, DE 19 DE FEVEREI- } \\
\text { RO DE } 2002\end{array}$ & Não menciona & Não menciona \\
\hline Fonoaudiologia & $\begin{array}{c}\text { RESOLUÇÃO CNE/CES } \\
\text { 5, DE 19 DE FEVEREI- } \\
\text { RO DE } 2002\end{array}$ & Não menciona & Não menciona \\
\hline
\end{tabular}

\begin{tabular}{|c|c|c|c|}
\hline Medicina Veterinária & $\begin{array}{c}\text { RESOLUÇÃO CNE/CES } \\
\text { 1, DE } 18 \text { DE FEVEREI- } \\
\text { RO DE 2003.(*) }\end{array}$ & Não menciona & Não menciona \\
\hline Medicina & $\begin{array}{l}\text { RESOLUÇÃO CNE/ } \\
\text { CES No 3, DE } 20 \text { DE } \\
\text { JUNHO DE } 2014\end{array}$ & $\begin{array}{c}\text { Menciona, } \\
\text { Art. 5०: } \\
\text { Art. } 8^{\circ}: \\
\text { Art. 14:VI } \\
\text { Art. 17: } \\
\text { Art. 18: I } \\
\text { Art. } 24 \text { § } 3^{\circ} \S 4^{\circ} \\
\text { Art. 29. VII IX, } \\
\text { Art. 34: } \\
\text { Art. 35: }\end{array}$ & $\begin{array}{l}\text { Menciona, } \\
\text { Art. 5०: III } \\
\text { Art. 17: I }\end{array}$ \\
\hline Nutrição & $\begin{array}{l}\text { RESOLUÇÃO CNE/ } \\
\text { CES N 5, DE } 7 \text { DE NO- } \\
\text { VEMBRO DE } 2001\end{array}$ & $\begin{array}{l}\text { Menciona, } \\
\text { Art. } 5^{\circ} \text { : }\end{array}$ & Não menciona \\
\hline Odontologia & $\begin{array}{l}\text { RESOLUÇÃO CNE/CES } \\
\text { 3, DE 19 DE FEVEREI- } \\
\text { RO DE } 2002\end{array}$ & Não menciona & Não menciona \\
\hline Psicologia & $\begin{array}{l}\text { RESOLUÇÃO CNE/ } \\
\text { CES N 5, DE } 15 \text { DE } \\
\text { MARÇO DE } 2011\end{array}$ & Não menciona & $\begin{array}{l}\text { Menciona, } \\
\text { Art. 13: } § 2^{\circ}\end{array}$ \\
\hline Serviço Social & $\begin{array}{c}\text { RESOLUÇÃO CNE/CES } \\
\text { 15, DE } 13 \text { DE MARÇO } \\
\text { DE } 2002\end{array}$ & Não menciona & Não menciona \\
\hline Terapia Ocupacional & $\begin{array}{c}\text { RESOLUÇÃO CNE/CES } \\
\text { 6, DE 19 DE FEVEREI- } \\
\text { RO DE } 2002\end{array}$ & Não menciona & Não Menciona \\
\hline
\end{tabular}

Fonte: http://portal.mec.gov.br/component/content/article?id=12991. Acesso em 22 de março de 2018.(9)

Das 14 profissões em saúde, 10 (Biomedicina, Ciências Biológicas, Educação Física, Fisioterapia, Fonoaudiologia, Medicina Veterinária, Odontologia, Psicologia, Serviço Social e Terapia Ocupacional) não mencionam a palavra SUS em suas $\mathrm{DCN}$, enquanto que as políticas públicas também não foram mencionadas em 10 profissões (Biomedicina, Ciências Biológicas, Enfermagem, Fisioterapia, Fonoaudiologia, Medicina Veterinária, Nutrição, Odontologia, Serviço Social e Terapia Ocupacional).

O que mais chama a atenção é o fato de que nas DCN de oito profissões em saúde (Biomedicina, Ciências Biológicas, Fisioterapia, Fonoaudiologia, Medicina Veterinária, Odontologia, Serviço Social e Terapia Ocupacional) em nenhum momento é demonstrada a necessidade da inserção profissional no SUS e nas políticas públicas de saúde do Brasil.

Em observação às reformulações das DCN mais atuais, fica evidente que os cursos de Farmácia (DCN, 2017) e Medicina (DCN, 2014), articulam bem em suas competências e habilidades do futuro profissional essa dinâmica das polícias 
públicas promovida pelo SUS. Basta observar nas DCN do curso de Farmácia (Art. 4, Art. 5०, Art. $7^{\circ}$ e Art. 12\%), a descrição de que a formação do farmacêutico deve ser humanista, crítica, reflexiva e generalista, bem como pautar-se por uma concepção de referência nacional e internacional direcionando as instituições formadoras a nortear pelos princípios do SUS, como também pra a atuação profissional em conjunto com as políticas públicas e com o desenvolvimento científico e tecnológico, a fim de atender às necessidades sociais. Reforça essa habilidade no Art. 50, item XVII - prescrição, aplicação e acompanhamento das práticas integrativas e complementares inerentes à profissão, em consonância políticas públicas de saúde e a legislação vigente no Brasil nas diferentes instâncias.

As DCN do curso de Medicina, pautadas no princípio do profissional diretamente integrado às necessidades do SUS, faz a inserção longitudinal deste egresso.(10) Sendo que nos Art. $5^{\circ}, 8^{\circ}, 14^{\circ}, 17^{\circ}, 18^{\circ}, 24^{\circ}, 29^{\circ}, 34^{\circ}$ e $35^{\circ}$, ressaltam-se princípios e cenários inerentes ao SUS, seja no acompanhamento de pacientes, famílias ou em atividades de gestão e ensino, sedimentando conhecimentos, para a percepção da realidade na Saúde Pública, da responsabilidade profissional e social. Formar profissionais que saibam lidar com a população de forma ativa e resolutiva. Pelo desenho configurado nas DCN, nota-se que o enfoque permeia a Atenção Primária, permitindo que o estudante saiba gerir os agravos que possam atingir a população ${ }^{10}$.

No que abrange as políticas públicas, as DCN do curso de Medicina, nos Art. $5^{\circ}, 6^{\circ}$ e $17^{\circ}$, pautam um perfil profissional preocupado com a qualidade, gestão dos serviços de saúde, baseados nas evidências científicas, na escuta ativa e singular de cada pessoa, família, grupos e comunidades e nas políticas públicas, programas, ações estratégicas e diretrizes vigentes. Possibilitar ao profissional médico a tomada de decisões baseada em uma visão crítica e contextualizada das evidências científicas, enfim, que este profissional possa realizar a organização do trabalho em saúde, identificação da história da saúde, das políticas públicas de saúde no Brasil, da Reforma Sanitária e dos princípios do SUS ${ }^{10}$.

Entre as DCN dos cursos de Educação Física (DCN, 2004), Enfermagem (DCN, 2001) e Psicologia (DCN, 2011), somente a Enfermagem no Art. $5^{\circ}$, menciona que para a formação do Enfermeiro deve atender as necessidades sociais da saúde, com ênfase no Sistema Único de Saúde (SUS), enquanto que as DCN da Educação Física e Psicologia fazem referências às políticas públicas no Art. $6^{\circ}$ do curso de Educação Física, no qual recomenda a este profissional a operacionalização de políticas públicas para o SUS e institucionais nos campos da saúde e outras áreas. Já para o Psicólogo, as DCN em seu Art. $13^{\circ}$, faz referência em quatro momentos das políticas públicas, porém voltadas à área da educação, em nenhum momento voltando-se para a saúde ${ }^{10}$.

As DCN para os cursos de graduação da área da saúde são formadas por teorias e práticas pedagógicas, envolvidas ou não no processo de construção de um profissional a serviço da saúde da população como um todo. Acredita-se que a demanda pode ser atendida por elementos de uma teoria analítica das futuras matrizes curriculares destas profissões, desde que as DCN sofram reformulações e adequações, uma vez que, as escolas de saúde seguem em seus projetos pedagógicos curriculares, competências e habilidades listadas nas $\mathrm{DCN}^{11,12}$.

O Conselho Nacional de Saúde, em seu papel regulamentado pela constituição de 1988 questiona quais são os conhecimentos indispensáveis para que o futuro trabalhador em saúde possa entender e colocar em prática todo o conhecimento das políticas públicas e do SUS a favor da população. Essa realidade, que não está restrita à formação profissional, evidencia a necessidade dos profissionais de saúde compreender e praticar durante a graduação, as políticas públicas ofertadas pelo SUS, sobretudo no que diz respeito à estruturação de um sistema para todos, que atuem em contrapartida a um sistema capitalista que é imposto nas faculdades, ou seja, exigir formação profissional orientada por uma política pedagógica que entenda a saúde como universal ${ }^{13}$.

\section{Conclusão}

Este estudo, realizado em caráter investigativo, se limitou a uma análise das DCN que norteiam as 14 profissões da saúde no Brasil, sendo que a formação de um profissional voltado para a área da saúde extrapola a abordagem dessas diretrizes. Essa busca de inserção desperta uma reflexão crítica sobre a composição atual da maioria das DCN das profissões em saúde, as quais não contemplam as políticas públicas de saúde propostas pelo SUS.

Desta forma é possível que através da Resolução № 569 de 08 de dezembro de 2017, publicada pelo CNS, a concepção de Projetos Pedagógicos de Cursos (PPC) esteja alinhada aos problemas e às necessidades de saúde da população. Nesse sentido, cabe refletir que a atualização das DCN, com exceção dos cursos de Farmácia e Medicina, deve ser realizada o mais brevemente possível. É difícil para as Escolas de Ensino Superior buscar norteamento nas DCN atualizadas entre 2001 a 2004 (11 profissões). Do mesmo modo, no caso da Psicologia, apesar da última atualização ocorrida em 2011, suas DCN não contemplam a inserção do profissional nas políticas públicas do SUS e também não articulam a formação com o cotidiano dos serviços de saúde em seus diversos níveis de atenção. 
A aplicação das políticas públicas do SUS nas DCN dos cursos de graduação da área da saúde tem por objetivo modificar currículos e programas, fazendo uma contextualização do que está previsto nas diretrizes para os processos de formação das escolas no ensino e aprendizagem. Expressa-se bem essa ideia de inserção das políticas públicas e do SUS nas DCN dos cursos voltados para a saúde: "Fecha se, com isso, um ciclo móvel da estrutura de ensino superior na área da saúde, no qual se inserem instituições da área da educação: do SUS às DCN, das DCN aos cursos de graduação, dos cursos aos alunos. Transformados em profissionais, eles ingressam no trabalho com as competências desenvolvidas nos processos de formação"14.

Diante deste contexto, mudanças são necessárias para possibilitar a construção de estruturas curriculares que compõe os PPC, no sentido de formar profissionais com as competências e as habilidades pertinentes às políticas públicas praticadas na constituição SUS.

\section{Referências Bibliográficas}

1. Brasil. Constituição de 1988. Constituição da República Federativa do Brasil. Brasília. DF: Senado, 2014.

2. Bertollini Filho C. A história da Saúde Pública no Brasil. São Paulo: Ática, 2001.

3. Lucchese PTR. (coord.). Políticas Públicas em Saúde Pública. São Paulo: IBIREME/OPAS/OMS, 2002.

4. Roncalli AG. O desenvolvimento das políticas de saúde no Brasil e a construção do Sistema Único de Saúde. In: Pereira AC. Odontologia em saúde coletiva: planejando ações e promovendo saúde. Porto Alegre: Artmed, 2003.

5. Paulus Júnior A, Cordoni Júnior L. Políticas Públicas de Saúde no Brasil. Revista Espaço para a Saúde, 2006;8(1):13-19.

6. Brasil. Ministério da Saúde. Conselho Nacional de Secretárias Municipais de Saúde. O SUS de A a Z: garantindo saúde nos municípios. 3. ed. 2. reimp. Brasília: Ministério da Saúde, 2009. (Série F. Comunicação e Educação em Saúde). Disponível em: . Acesso em: 20 mar. 2018.

7. Costa NMSC. Docência no ensino médico: por que é tão difícil mudar? Rev Bras Educ Med 2007;31(1):21-30.

8. Conselho Nacional de Saúde (CNS). http://conselho.saude. gov.br/resolucoes/2017/ Reso569.pdf > acesso em 10 de abril de 2018.

9. Ministério da Educação (MEC). <http://portal.mec.gov.br/ component/content/article ?id=1299 1> Acesso em 22 de março de 2018

10. Siqueira-Batista R, Gomes AP, Albuquerque VS, Cavalcanti FOL, Cotta RMM. Educação e competências para o SUS: é possível pensar alternativas à(s) lógica(s) do capitalismo tardio? Ciências Saúde Colet. 2013; 18(1):159-70.
11. Almeida Filho N. Reconhecer Flexner: inquérito sobre produção de mitos na educação médica no Brasil contemporâneo. Cadernos de Saúde Pública. 2010;26(12):2234-49.

12. Nunes ED, Nascimento JL, Barros NF. A questão curricular para o plano de formação em Saúde Coletiva: aspectos teóricos. Ciências Saúde Colet. 2010;15(4):1935-43.

13. Almeida Filho NM. Contextos, impasses e desafios na formação de trabalhadores em Saúde Coletiva no Brasil. Ciências Saúde Colet. 2013;18(6):1677-82.

14. Moreira COF, Araújo D. Diretrizes Curriculares na saúde e as mudanças nos modelos de saúde e de educação. Arquivos Brasileiros de Ciências da Saúde. 2015;40(3):300-5. 